\title{
Selecting Optimal Portfolio in Generalised Feed Forward Networks And Self Organized Features Maps Hybrids
}

\author{
N., Loukeris \\ Athens University of \\ Economics and Business \\ Department of \\ Accounting \& Finance \\ Patision 76, Athens, \\ Greece \\ nloukeris@uom.edu.gr
}

\author{
I., Eleftheriadis \\ University of Macedonia \\ Interdepartmental \\ Program of Postgraduate \\ Studies in Information \\ Systems \\ \& \\ Dept. of Business \\ Administration \\ Egnatias 156, \\ Thessaloniki, Greece \\ jordan@uom.gr
}

\author{
E., Kokkinos \\ Hellenic Mediterranean \\ University \\ Department
}

of Electronics Engineers

Romanou 3, Chania

73100, Crete, Greece

ekokkinos@hmu.gr

Received: July 7, 2020. Revised: September 18, 2020. Accepted: September 28, 2020. Published: September 30, 2020.

\begin{abstract}
We evaluate the performance of 70 Generalised Feed Forward and 60 Self Organized Feature Maps models of plain and hybrid form to define the optimal classifier in portfolio selection. We also apply it on a novel model of optimal portfolio selection in hedging aspects.
\end{abstract}

Index Terms — Genetic Algorithms, Generalised Feed Forward, Hybrid Networks, Self Organized Feature Maps, Hedge
Management, Portfolio Optimization

\section{INTRODUCTION}

The 2 phase process of portfolio selection advanced to detailed aspects of risk in further higher moments (volatility, hyperkurtosis,

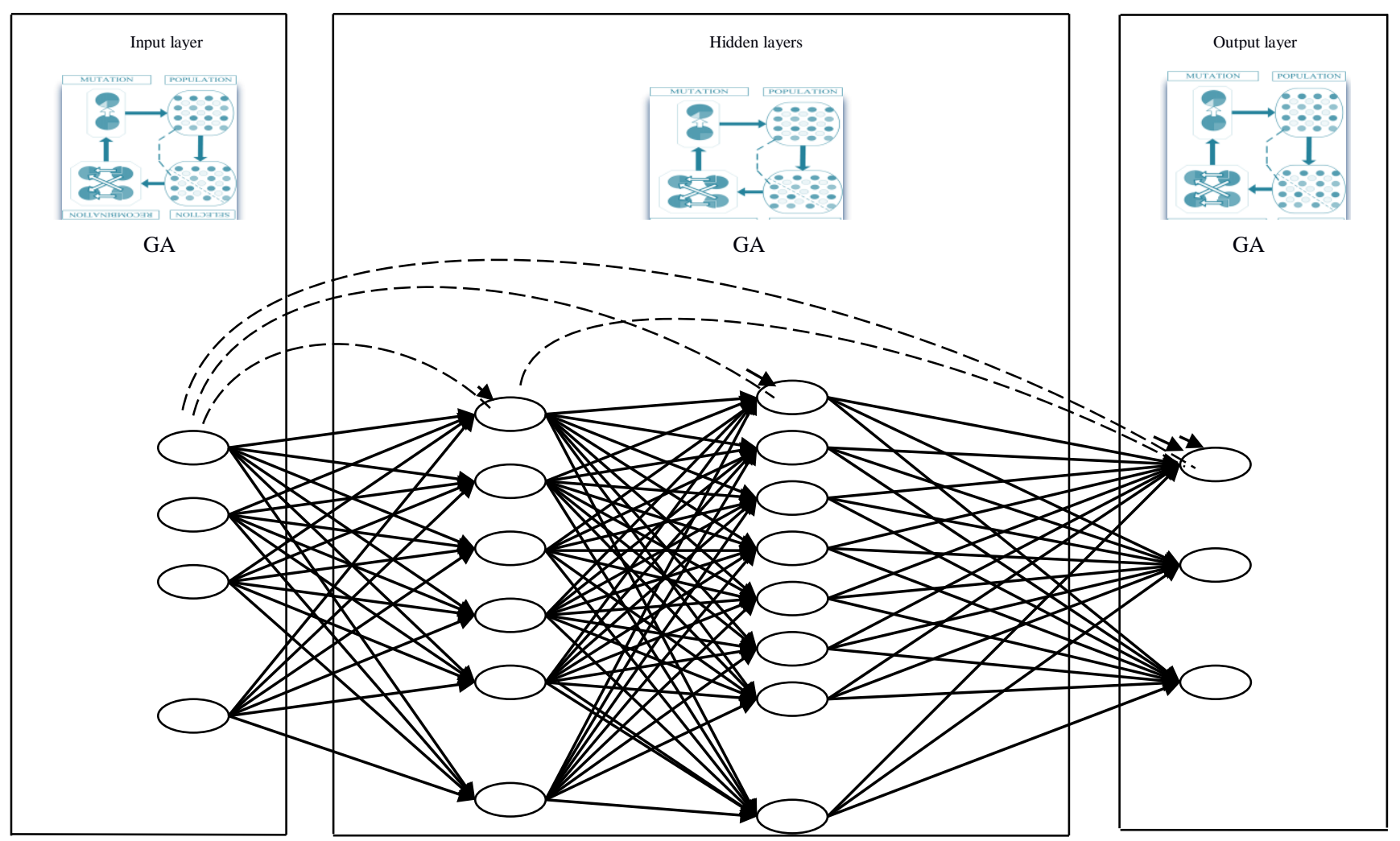

Figure 1. The Hybrid Generalised Feed Forward networks, of GA optimization in all layers and CV.

ultrakurtosis, hyperultrakurtosis, etc), Loukeris and Eleftheriadis (2017) is developed in this paper. On the first step portfolios are evaluated, forming a feasible set, and secondly the ranked efficient portfolios minimize the risk on a utility function, Loukeris and
Eleftheriadis (2020, 2019, 2017, 2016, 2015, 2014, 2012), Loukeris et al. (2009), Loukeris (2006, 2008). This article evaluates the first step that resolves the second step. We thoroughly examine the systems of neural or neuro-genetic 
hybrids: 70 Generalised Feed Forward and 60 different Self Organized Feature Maps networks in 20 neural networks and 50 GFF hybrids as 20 neural nets and 40 hybrid neuro-genetic SOFM models of alternative topologies that seek the most efficient neural model to classify the portfolio selection.

\section{THE HYBRID GENERALISED FEED FORWARD NETWORKS}

The Generalised Feed Forward networks, (figure 1) are a generic form of the Multi Layer Perceptrons that are able to let their synapses jump over one or more layers. Into the GFFs we apply an initial MLP as in each layer its signals feeds forward all the forthcoming layers. The actual performance of the GFFs revealed that they are much more efficient resolving classifiers to the problem than the MLPs, in much shorter time, for a similar number of neurons.

\section{SELF ORgANIZED FEATURES MAPS}

The Self Organized Features Maps-SOFM neural network, Kohonen (1982), or Kohonen map, is optimized for clustering, and data examination. The SOFM is trained in unsupervised learning forming a two-dimensional map. This discretised input surface of the training set reduces dimensionality. The SOFMs use competitive learning, alternating from the other neural nets. The 16 financial indices have an unknown significance in the SOFM nets and we incorporate Genetic Algorithms-GA, Holland (1975/1992), to determine it. Each model is trained multiply to conclude in the inputs of the lowest error. The GAs are elaborated in different hybrids both for the MLPs and SOFMs of the: i) inputs layer only, ii) inputs and outputs layers only, iii) all layers and cluster centers, iv) all layers and cluster centers with cross validation, in different topologies. The Batch learning adjusts the weights of hybrid SOFM.

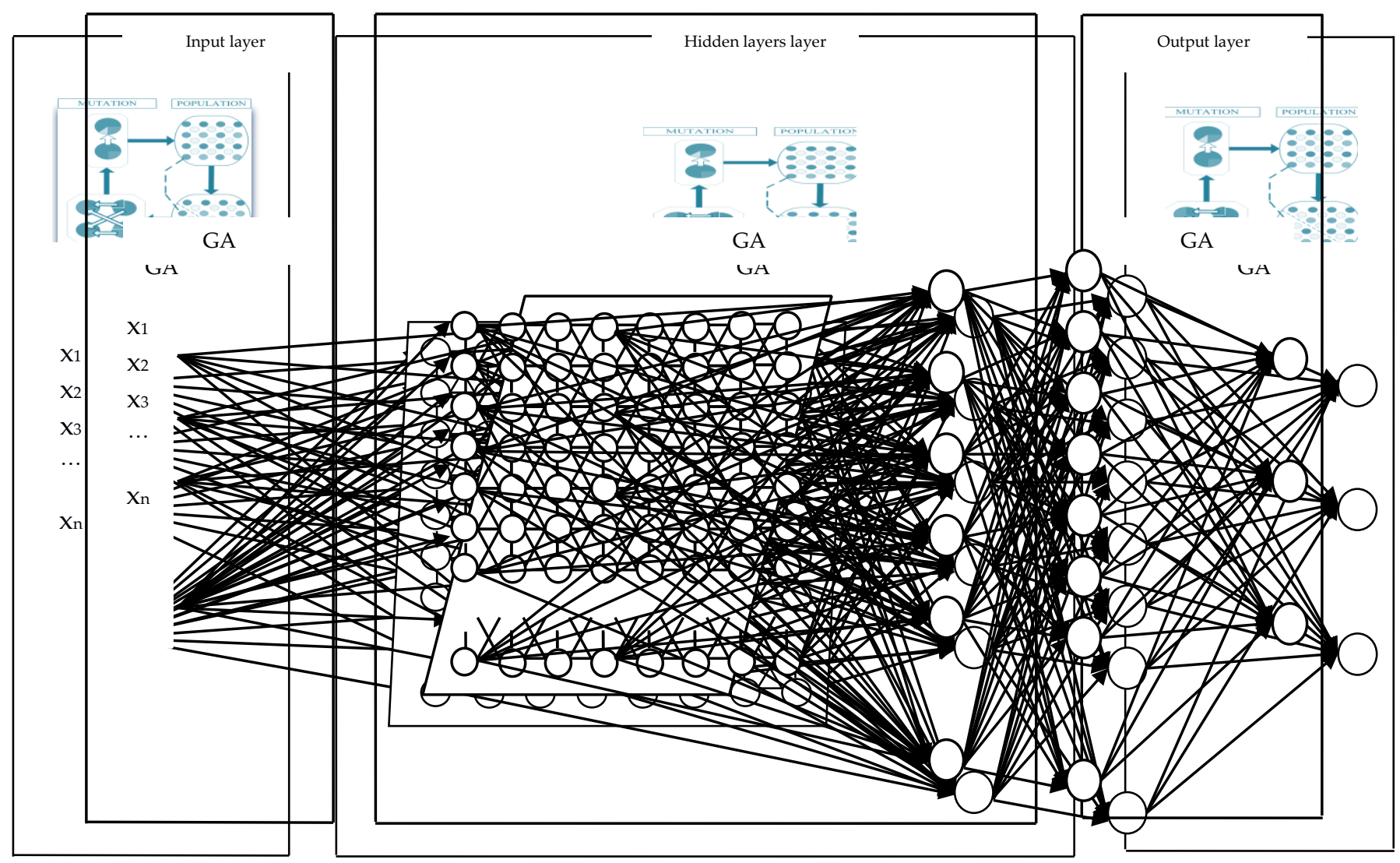

Figure 2. Hybrid Self Organized Feature Maps with Genetic optimization on all layers and Cross Validation, Loukeris, Chalamandaris, Eleftheriadis (2019),

\section{DATA OF NEURAL COMPUTATION}

The data came by 1411 companies from the loan department of a Greek commercial bank, with the following 16 financial indices: 1) EBIT/Total Assets, 2) Net Income/Net Worth, 3) Sales/Total Assets, 4) Gross Profit/Total Assets, 5) Net Income/Working Capital, 6) Net Worth/Total Liabilities, 7) Total Liabilities/Total assets, 8) Long Term Liabilities /(Long Term Liabilities + Net Worth), 9) Quick Assets/Current Liabilities, 10) (Quick AssetsInventories)/Current Liabilities, 11) Floating Assets/Current Liabilities, 12) Current Liabilities/Net Worth, 13) Cash Flow/Total Assets, 14) Total Liabilities/Working Capital, 15) Working Capital/Total Assets, 16) Inventories/Quick Assets, and a 17th index with initial classification, by bank experts Courtis (1978). The test set was $50 \%$ of overall data, and the training $50 \%$. The 1411 companies are unique, the observation has discrete frequency in 3 different annual values and the average is implemented, Courtis (1978), the dependent value $\varepsilon_{\tau}$ is binary, in 0 for the healthy, and 1 for the distressed companies. The classification process Kohonen (1982) of the SOFM models is processed by the appropriate classifier, Principe, deVries, Kuo and Oliveira (1992), Principe, Euliano, and Lefebvre, (1999). 
IV. RESUlts OF THE GENERALISED FEED FORWARD MODELS The GFF hybrid of 1 layer in Genetic Algorithms optimisation on the inputs and outputs layers only was the optimal performance classifier in high correct classifications of the healthy and the distressed companies at $98.9 \%$ and $88.52 \%$ respectfully, very high fitness of the model to the data as $\mathrm{r}$ was 0.908 , the lowest error as MSE was 0.072, NMSE 0.170 and percentage error 5.77, whilst the AIC was very low at -1907.09 indicating impartiality and a time of $3 \mathrm{~h} 19 \mathrm{~m} 25 \mathrm{~s}$.
The next rank was taken by the hybrid GFF of 3 layers in GA optimization in all layers, in an almost fine classification, a high fitness of the model to the data at $\mathrm{r}$ 0.834, low error, and impartiality in $4 \mathrm{~h} 20 \mathrm{~m} 25 \mathrm{~s}$.

A slightly inferior performance had the hybrid GFF of 1 layer and GA optimization in all layers, in terms of classification, fitness, and error, whilst the impartiality was higher, in a time of $3 \mathrm{~h} 19 \mathrm{~m}$ 25s Loukeris and Eleftheriadis (2016).

Table 1. Ranking of the optimal GFF models overall, Loukeris and Eleftheriadis (2016)

\begin{tabular}{|c|c|c|c|c|c|c|c|c|c|c|c|c|}
\hline \multicolumn{2}{|l|}{ Models } & \multicolumn{4}{|c|}{ Active Confusion Matrix } & \multicolumn{7}{|c|}{ Performance } \\
\hline & & $\mathbf{0} \rightarrow \mathbf{0}$ & $\mathbf{0} \rightarrow \mathbf{1}$ & $1 \rightarrow 0$ & $1 \rightarrow 1$ & MSE & NMSE & $\mathbf{r}$ & \%error & AIC & MDL & Time \\
\hline GFF input-output GA & 1 & 98.90 & 1.085 & 11.465 & 88.52 & 0.072 & 0.170 & 0.908 & 5.776 & -1907.09 & -1796.44 & $3 \mathrm{~h} 19^{\prime} 25^{\prime \prime}$ \\
\hline GFF GA all & 3 & 97.14 & 2.845 & 17.885 & 82.10 & 0.128 & 0.304 & 0.834 & 8.343 & -786.38 & 284.34 & $4 h 20^{\prime} 25^{\prime \prime}$ \\
\hline GFF GA all & 1 & 97.56 & 2.425 & 18.805 & 81.18 & 0.133 & 0.315 & 0.827 & 8.243 & -723.47 & -271.82 & $3 \mathrm{~h} 19^{\prime} 25^{\prime \prime}$ \\
\hline GFF GA all, & 7 & 96.64 & 3.35 & 19.26 & 80.73 & 0.136 & 0.323 & 0.825 & 9.119 & 1541.07 & 3429.31 & $25 \mathrm{~h} 46^{\prime} 34^{\prime \prime}$ \\
\hline $\mathrm{CV}$ & & 98.32 & 1.67 & 29.355 & 70.63 & 0.149 & 0.353 & 0.812 & 7.023 & 1608.29 & 3495.49 & \\
\hline GFF NN & 1 & 97.73 & 2.26 & 21.095 & 78.89 & 0.138 & 0.328 & 0.821 & 9.675 & -1225.82 & -1111.95 & 14 ', \\
\hline GFF NN, CV & 8 & 98.23 & 1.755 & 26.14 & 73.85 & 0.143 & 0.338 & 0.814 & 9.284 & 709.44 & 2041.35 & $42.5^{\prime}$ \\
\hline $\mathrm{CV}$ & & 98.23 & 1.755 & 26.14 & 73.85 & 0.143 & 0.338 & 0.814 & 9.284 & 709.44 & 2041.35 & \\
\hline GFF GA inputs & 10 & 97.98 & 2.005 & 26.6 & 73.16 & 0.144 & 0.341 & 0.812 & 9.469 & 1219.39 & 2873.69 & $7 \mathrm{~h} 44^{\prime} 32^{\prime \prime}$ \\
\hline GFF GA all & 8 & 98.57 & 1.42 & 26.6 & 73.39 & 0.140 & 0.329 & 0.821 & 8.329 & 1262.65 & 2959.69 & $29 \mathrm{~h} \mathrm{50'17^{ \prime \prime }}$ \\
\hline GFF GA all, CV & 1 & 97.98 & 2.005 & 24.305 & 75.68 & 0.145 & 0.343 & 0.810 & 8.646 & -1219.07 & -1126.3 & $2 \mathrm{~h} 27^{\prime} 41^{\prime \prime}$ \\
\hline $\mathrm{CV}$ & & 98.4 & 1.59 & 24.765 & 75.22 & 0.139 & 0.330 & 0.821 & 8.686 & -1242.55 & -1149.79 & \\
\hline GFF NN & 10 & 98.65 & 1.34 & 31.185 & 68.80 & 0.147 & 0.348 & 0.811 & 8.454 & 1557.50 & 3419.165 & $57^{\prime \prime}$ \\
\hline
\end{tabular}

\section{RESULTS OF THE SOFM MODELS}

The optimal overall classifier was the Hybrid SOFM of 2 layers GA optimization in all layers and Cross Validation, in $98.82 \%$ and $59.63 \%$ correct classification of the healthy and distressed companies that produces an acceptable classification, a medium fitness to the model $\mathrm{r}$ at 0.656 , an average level of error, significant partiality in $740.06 \mathrm{AIC}$, requiring a significant 2 hours 35 minutes and 29 seconds of processing time.

Second was ranked the SOFM hybrid with GAs that optimized all layers, medium classification in $99.07 \%$ and $36.23 \%$ of the healthy and distressed firms, a medium fitness to the model at 0.614, medium error, higher partiality at 1290.15 AIC, and higher time of 2 hoursa 26 minutes 13 seconds.

Third was the SOFM hybrid with genetic optimization in the inputs and output layers only of 2 layers, in $97.57 \%$ and $43.16 \%$ correct classifications of the healthy and distressed companies, a medium fitness of the model to the data at 0.581, slightly lower error, an Akaike at 389.25 indicating a significant partiality, with a lower time of 1 hour and 15 ' 43 ',.

Table 2. Ranking of the optimal SOFM models overall

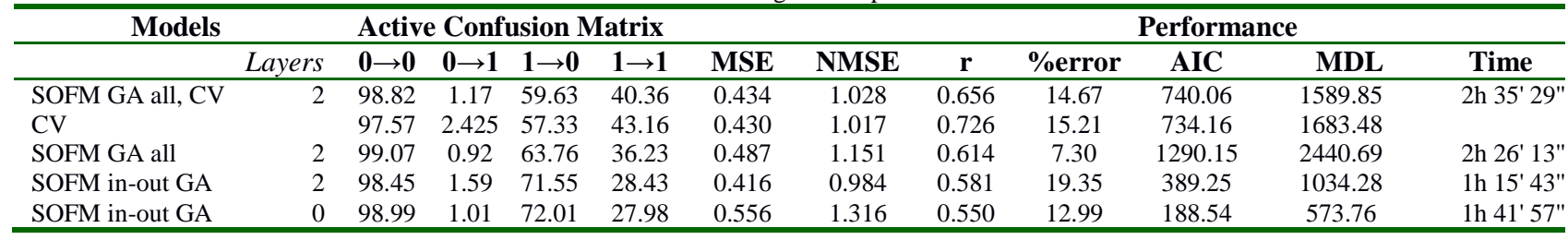

\section{CONCLUDING REMARKS}

The GFF hybrids had a significantly superior performance than the SOFMs.

Specifically the overall optimal classifier between the examined groups of GFFs, and SOFMs was the GFF Hybrid of GA optimization on the input and output layers only, with 1 hidden layers, very good classification, and fitness, low error, impartiality and low computing time.

The second overall best classifier was the GFF Hybrid of GA optimization in all layers, with 3 hidden layers, very good classification, and fitness, low error, impartiality and higher time. Third overall classifier was the GFF of GAs in all layers and Cross Validation, in 1 hidden layer, very good classification and fitness as well, lower error, impartiality and medium time.

The SOFMs underperformed in all cases and were ranked between the positions from $11^{\text {th }}$ to $13^{\text {th }}$ in grounds of optimal performance.
Unfortunately the best SOFM model had the 11 rank of lower classification, medium fitness and higher error, in a shorter computational time. Hence we notice that the simple MLP core with feedforward signal performs betters than the Kohonen architecture in this data classification case. The simple design where the synapses jump over one or more layers of GFs is more efficient than the two dimensional maps and the competitive learning process of the Kohonen core in the SOFMs. Even the evolutional computing alternative of Genetic Algorithms couldn't provide a robust competitive advantage to the Kohonen approach. In our case a simple hybrid of feedforward process with minimal evolutional support was the most effective solution in the specific problem. 
Table 3. Overall ranking of the optimal GFF and SOFM models

\begin{tabular}{|c|c|c|c|c|c|c|c|c|c|c|c|c|}
\hline \multicolumn{2}{|l|}{ Models } & \multicolumn{4}{|c|}{ Active Confusion Matrix } & \multicolumn{7}{|c|}{ Performance } \\
\hline & ers & $\mathbf{0} \rightarrow \mathbf{0}$ & $\mathbf{0} \rightarrow \mathbf{1}$ & $\mathbf{1} \rightarrow \mathbf{0}$ & $1 \rightarrow 1$ & MSE & NMSE & $\mathbf{r}$ & \%error & AIC & MDL & Time \\
\hline GFF input-output GA & 1 & 98.90 & 1.085 & 11.465 & 88.52 & 0.072 & 0.170 & 0.908 & 5.776 & -1907.09 & -1796.44 & $3 h 1^{\prime} 25^{\prime \prime}$ \\
\hline GFF GA all & 3 & 97.14 & 2.845 & 17.885 & 82.10 & 0.128 & 0.304 & 0.834 & 8.343 & -786.38 & 284.34 & $4 \mathrm{~h} 20^{\prime} 25^{\prime \prime}$ \\
\hline GFF GA all & 1 & 97.56 & 2.425 & 18.805 & 81.18 & 0.133 & 0.315 & 0.827 & 8.243 & -723.47 & -271.82 & $3 \mathrm{~h} 19^{\prime} 25^{\prime \prime}$ \\
\hline GFF GA all, & 7 & 96.64 & 3.35 & 19.26 & 80.73 & 0.136 & 0.323 & 0.825 & 9.119 & 1541.07 & 3429.31 & $25 \mathrm{~h} 46^{\prime} 34^{\prime \prime}$ \\
\hline $\mathrm{CV}$ & & 98.32 & 1.67 & 29.355 & 70.63 & 0.149 & 0.353 & 0.812 & 7.023 & 1608.29 & 3495.49 & \\
\hline GFF NN & 1 & 97.73 & 2.26 & 21.095 & 78.89 & 0.138 & 0.328 & 0.821 & 9.675 & -1225.82 & -1111.95 & $14{ }^{\prime}$ \\
\hline GFF NN, CV & 8 & 98.23 & 1.755 & 26.14 & 73.85 & 0.143 & 0.338 & 0.814 & 9.284 & 709.44 & 2041.35 & $42.5^{\prime}$ \\
\hline $\mathrm{CV}$ & & 98.23 & 1.755 & 26.14 & 73.85 & 0.143 & 0.338 & 0.814 & 9.284 & 709.44 & 2041.35 & \\
\hline GFF GA inputs & 10 & 97.98 & 2.005 & 26.6 & 73.16 & 0.144 & 0.341 & 0.812 & 9.469 & 1219.39 & 2873.69 & $7 \mathrm{~h} 44^{\prime} 32^{\prime \prime}$ \\
\hline GFF GA all & 8 & 98.57 & 1.42 & 26.6 & 73.39 & 0.140 & 0.329 & 0.821 & 8.329 & 1262.65 & 2959.69 & $29 \mathrm{~h} \mathrm{50'17^{ \prime \prime }}$ \\
\hline GFF GA all, CV & 1 & 97.98 & 2.005 & 24.305 & 75.68 & 0.145 & 0.343 & 0.810 & 8.646 & -1219.07 & -1126.3 & $2 \mathrm{~h} 27^{\prime} 41^{\prime \prime}$ \\
\hline $\mathrm{CV}$ & & 98.4 & 1.59 & 24.765 & 75.22 & 0.139 & 0.330 & 0.821 & 8.686 & -1242.55 & -1149.79 & \\
\hline GFF NN & 10 & 98.65 & 1.34 & 31.185 & 68.80 & 0.147 & 0.348 & 0.811 & 8.454 & 1557.50 & 3419.165 & $57^{\prime}$ \\
\hline SOFM GA all, CV & 2 & 98.82 & 1.17 & 59.63 & 40.36 & 0.434 & 1.028 & 0.656 & 14.67 & 740.06 & 1589.85 & $2 h^{\prime} 35^{\prime} 29^{\prime \prime}$ \\
\hline $\mathrm{CV}$ & & 97.57 & 2.425 & 57.33 & 43.16 & 0.430 & 1.017 & 0.726 & 15.21 & 734.16 & 1683.48 & \\
\hline SOFM GA all & 2 & 99.07 & 0.92 & 63.76 & 36.23 & 0.487 & 1.151 & 0.614 & 7.30 & 1290.15 & 2440.69 & $2 \mathrm{~h} 26^{\prime} 13^{\prime \prime}$ \\
\hline SOFM in-out GA & 2 & 98.45 & 1.59 & 71.55 & 28.43 & 0.416 & 0.984 & 0.581 & 19.35 & 389.25 & 1034.28 & 1h $15^{\prime} 43^{\prime \prime}$ \\
\hline
\end{tabular}

\section{REFERENCES}

[1]Courtis J., (1978), Modelling a Financial Ratios Categoric Framework, Journal of Business Finance \& Accounting, 5, 371386 ,

[2]Holland, J. H., (1975/1992), Adaptation in Natural and Artificial Systems. Cambridge, MA, MIT Press, Second edition (1992). (First edition, University of Michigan Press)

[3]Kohonen, T., (1982), Self-Organized Formation of Topologically Correct Feature Maps, Biological Cybernetics, 43 (1): 59-69,

[4]Loukeris, N., and N. Matsatsinis, (2006a), Corporate Financial Evaluation and Bankruptcy Prediction implementing Artificial Intelligence methods, WSEAS Trans. Business and Economics, $4(3)$,

[5]Loukeris N., (2008), Comparative Evaluation of Multi Layer Perceptrons to hybrid MLPs with Multicriteria Hierarchical Discrimination and Logistic Regression in Corporate Financial Analysis, $11^{\text {th }}$ International Conference on Computers CSCC, Elounda Agios Nikolaos, Crete, Greece 26-28 July

[6]Loukeris, N., D. Donelly, A. Khuman, Y. Peng, (2009), A numerical evaluation of meta-heuristic techniques in Portfolio Optimisation, Operational Research, Volume 9 (1), ed. Springer,

[7]Loukeris N., and I.Eleftheriadis, (2012a), Bankruptcy Prediction into Hybrids of Time Lag Recurrent Networks with Genetic optimisation, Multi Layer Perceptrons Neural Nets, and Bayesian Logistic Regression, Proc. Int.Summer Conference of the International Academy of Business and Public Administration Disciplines (IABPAD), Honolulu, Hawaii, USA (August 1- 5) Research Paper Award

[8]Loukeris N., Eleftheriadis I., and S. Livanis (2014a) Optimal Asset Allocation in Radial Basis Functions Networks, and hybrid neuro-genetic RBFNs to TLRNs, MLPs and Bayesian Logistic Regression, World Finance Conference, July 2-4, Venice, Italy [9]Loukeris N., Eleftheriadis I. and E. Livanis (2014b), Portfolio Selection into Radial Basis Functions Networks and neuro-genetic RBFN Hybrids, IEEE $5^{\text {th }}$ Int.Conf. IISA, July 7-9, Chania Greece, [10]Loukeris N. and I. Eleftheriadis, (2015b), Further Higher Moments in Portfolio Selection and A-priori Detection of Bankruptcy, under Multi Layer Perceptron Neural Networks, Hybrid Neuro-Genetic MLPs, and the Voted Perceptron, International Journal of Finance and Economics, 20(4), October, ed. Wiley co,
[11]Loukeris N., and Eleftheriadis I., (2016), Generalized FeedForward Hybrids in Portfolio Selection, 2016 International Conference on Computational Science and Computational Intelligence, CSCI'16, December 15-17, Las Vegas, USA

[12]Loukeris N., I. Eleftheriadis, (2017), Control of Corporate Ownership in the Evolutional Portfolio Intelligent Complex Optimization (EPICO) model, Corporate Ownership and Control 14(4-1), 271-285,

[13]Loukeris N., Chalamandaris G., Eleftheriadis I. (2019), Self Organized Features Maps SOFM and hybrid neuro-genetic SOFMs in optimal portfolio management, IEEE 2019 International Conference on Computational Science and Computational Intelligence, CSCI 2019, Symposium on Artificial Intelligence (CSCI-ISAI) 5-7, December, Las Vegas, Nevada, USA

[14]Loukeris N., Eleftheriadis I. (2020), Optimal Portfolio Selection in MultiLayer Perceptrons and Self Organized Features Maps hybrids, 2020 International Symposium on Business and Management (ISBM 2020), 1-3 April, Sydney, Australia

[15]Maringer D., and P. Parpas, (2009), Global Optimization of Higher Order Moments in Portfolio Selection, J. Global Optimization. (43)2-3,

[16]Principe J., deVries B., Kuo J. and Oliveira P., (1992), Modeling applications with the focused gamma network, Neural Information Processing Systems 4, (eds. Moody, Hanson, Touretsky), pp121-126, Morgan Kaufmann,

[17]Principe J., Euliano R., and C., Lefebvre, (1999), Neural and adaptive systems: Fundamentals through simulations, Wiley NY

[18]Rumelhart D., G. Hinton, \& R. Williams, (1986), Learning internal representations by error back-propagation, in Parallel distributed processing: explorations in the microstructure of cognition (Rumelhart, D. \& McClelland, J., eds.), MIT Press, Cambridge, $M A$.

[18]Rua, A. and Nunes, L. (2009) International Co-Movement of Stock Market Returns: A Wavelet Analysis. Journal of Empirical Finance, 16, 632-639.

[19]Vacha, L. \& Barunik, J. (2012), Comovement of energy commodities revisited evidence from wavelet coherence analysis, Energy Economics 34, 241-247

Vacha, L., Janda, K., Kristoufek, L. \& D., Zilberman, (2013), Time-frequency dynamics of biofuel-fuel-food system, Energy Economics, 40, 233-24

\section{Creative Commons Attribution License 4.0 (Attribution 4.0 International, CC BY 4.0)}

This article is published under the terms of the Creative Commons Attribution License 4.0

https://creativecommons.org/licenses/by/4.0/deed.en_US 